Pacific Journal of Mathematics

A MODULI REPRESENTATION FOR THE CLASSIFICATION

Elyahu Katy 


\title{
A MODULI REPRESENTATION FOR THE CLASSIFICATION OF TWISTED TENSOR PRODUCTS
}

\author{
ELyahu Katz
}

\begin{abstract}
A differential Lie algebra $L(B, A)$ is associated to a differential graded coalgebra $B$ and differential graded algebra $A$. Taking the quotient of the "integrable" elements $I(B, A)$ of $L(B, A)^{1}$ by the action of $L(B, A)^{0}$ via the exponential map exp, we get a moduli space $E(B, A)=I(B, A) / \exp L(B, A)^{0}$ which represents the equivalence classes of $(B, A)$ twisted tensor products. For computations we may apply methods of algebraic geometry. This is applied to find an approximation for the cardinality of equivalence classes of Serre fibrations.
\end{abstract}

1. Introduction. To classify $(B, A)$ twisted tensor products, we introduce a differential graded Lie algebra $L(B, A)$, which consists of the $(B, A)$ twisting cochains. The equivalence classes of twisted tensor products turn out to be isomorphic to the orbit space of the integrable elements in $L(B, A)$ by conjugation with the automorphisms of $B \otimes A$ which preserve the extended module and comodule structures of $B \otimes A$. So far all this can be done over any commutative ring. To apply methods of algebraic geometry, we have to restrict our attention to field coefficients of characteristic 0 .

In $\S 2$ we prove that the bundles are the skeleton of the category of twisted tensor products, and also motivate the construction of the Lie algebra in $\S 3$ and the classification of twisted tensor products in $\S 4$. In $\S 5$ we introduce the classifying moduli space and $\S 6$ is devoted to stating some relevant algebraic geometry theorems and addaptations to our situation. The last two sections concern the approximation of fibrations by twisted tensor products. The basic references for this paper are: Schlessinger-Stasheff [6] and Gugenheim [3].

I would like to thank Professor James Stasheff for introducing me to methods in algebraic topology which involve Lie algebras and Algebraic Geometry, for suggesting the problem and for going over the first draft of this paper. I am indebted to Professor Michael Schlessinger for useful discussions on Algebraic Geometry. I would like also to thank Professor Jefferey Caruso for useful discussions with regard to this paper.

2. The relation between bundles and twisted tensor products. In this section we consider principal bundles and twisted tensor products and relate them to each other in a way that was done by 
Gugenheim [3]. While there mainly the structure of the bundles and the twisted tensor products was considered, here we also focus on the morphisms. We prove that the bundles are the skeleton of the category of twisted tensor products.

We denote by $\mathscr{A}$ the category of connected differential graded augmented algebras, and by $\mathscr{B}$ the category of connected differential graded augmented coalgebras.

Definition 1 . We denote by $\mathscr{B} \mathscr{C} \mathscr{A}$ the category of principal bundles. An object $(M, D, \phi, \psi)$ is a connected differential graded module such that ignoring differentials it is $B \otimes A$ with extended right $A$ module structure $\phi$ and extended left $B$ comodule structure $\psi$. We also require the following diagram to commute:

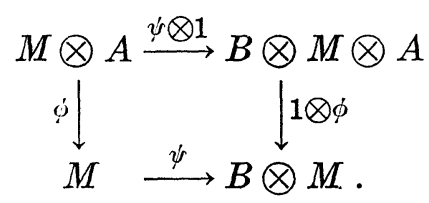

We simplify this notation and call it a $(B, A)$ bundle $M$.

A morphism in the category $(F, \beta, \alpha): M \rightarrow M^{\prime}$ has to make the following diagrams commutative:

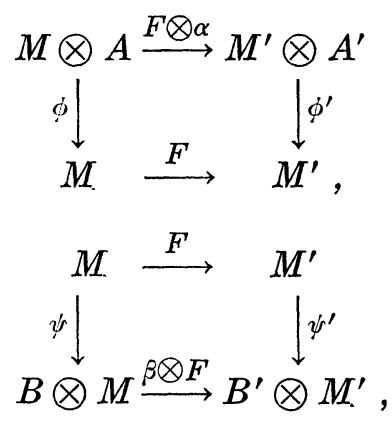

where $F: M \rightarrow M^{\prime}$ is a differential graded map, $\alpha \in \mathscr{A}$ and $\beta \in \mathscr{B}$.

Next we introduce the elements which make up the category of twisted tensor products.

Let $f: B \rightarrow A^{\prime}$ be a graded linear function of degree $k$. We define $f \cap: B \otimes A \rightarrow B^{\prime} \otimes A^{\prime}$ by $f \cap=\left(1 \otimes \dot{\phi}^{\prime}\right)(\beta \otimes f \otimes 1)(\psi \otimes \alpha)$. This is defined for $\alpha \in \mathscr{A}$ and $\beta \in \mathscr{B}$. Of course the resulting map $f \cap$ is also of degree $k$. Note that we use here $\phi$ and $\psi$ as the algebra structure of $A$ and the coalgebra structure of $B$ respectively. The context will always make it possible to distinguish this from the module and comodule structures of a $(B, A)$ bundle.

Proposition 1. ([3]) Let $(M, D) \in \mathscr{B} \mathscr{A l} \mathscr{A}$. Then $D=D_{0}+x \cap$, 
where $D_{0}=d \otimes 1+1 \otimes d, x$ is a degree -1 linear map $x: B \rightarrow A$ defined by $x=(\varepsilon \otimes 1) D(1 \otimes \eta), \varepsilon$ and $\eta$ are the unit and counit of $A$ and $B$ respectively. $B \otimes A$ with this differential will be denoted by $B \bigotimes_{x} A$.

Proposition 2. Let $(F, \beta, \alpha): M \rightarrow M^{\prime}$ be a morphism in the

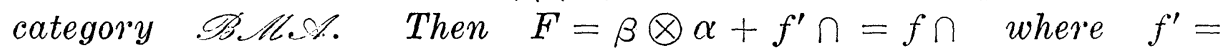
$\left(\varepsilon^{\prime} \otimes 1\right)(F-\beta \otimes \alpha)(1 \otimes \eta)$, and $f=\left(\varepsilon^{\prime} \otimes 1\right) F(1 \otimes \eta)$.

REMarks 1. The way we prove Proposition 2 (see below) can be modified to a proof of Proposition 1 which was originally proved differently.

2. A slight modification of the proof of Proposition 2 also proves the following: Let $M, M^{\prime}$ be elements of $\mathscr{B} \mathscr{L} . M^{\prime}$ and $G: M \rightarrow M^{\prime}$ a map of degree $k \neq 0,-1$, such that when $F$ is replaced with $G$ in diagrams (2) and (3) we obtain commutativity. Then we have $G=g \cap$, with $g=\left(\varepsilon^{\prime} \otimes 1\right) G(1 \otimes \eta)$. Precisely maps of this form, $g \cap$, will be used in the sequel to form the Lie algebra we are interested in.

Proof of Proposition 2. Consider the following commutative diagram.

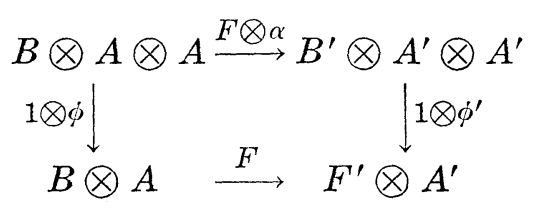

Applying commutativity on elements of the form $1 \otimes 1 \otimes a, b \otimes$ $1 \otimes a, b \otimes a_{1} \otimes a_{2}$ we obtain

$$
F(a)=\alpha(a), F(b \otimes a)=F(b) \alpha(a), F\left(b \otimes a_{1} a_{2}\right)=F\left(b \otimes a_{1}\right) \alpha\left(a_{2}\right) .
$$

We can restrict the diagram:

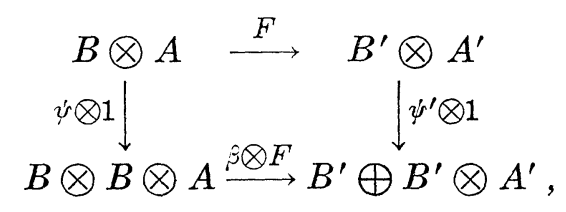

to the following one:

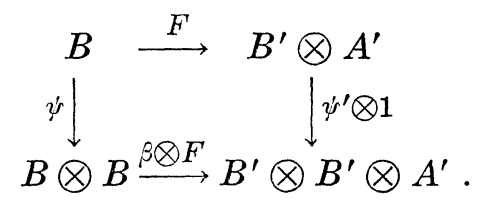


The latter diagram translates to the fact, that $F$ restricted to $B$ (which we also denote by $F$ ) is a comodule map from the $B$ comodule $B$ to the extended $B^{\prime}$ comodule $B^{\prime} \otimes A^{\prime}$. Because of the adjointness of the functors $V-\mid B \otimes(\quad)$, the forgetful and the extended comodule functors, we get:

$$
F=(\beta \otimes f) \psi=f \cap: B \longrightarrow B^{\prime} \otimes A^{\prime}
$$

where $f=\left(\varepsilon^{\prime} \otimes 1\right) F: B \rightarrow A^{\prime}$ [4]. Since $F(1)=1$ we get $f(1)=1$. Denote $f^{\prime}(b)=f(b)$ for $|b| \geqq 1$, and $f^{\prime}(1)=0(|b|=$ degree $b)$. Then

$$
F=\beta \otimes \alpha+f^{\prime} \cap \text {. }
$$

Now if we give $B \otimes A$ the filtration induced by the degree in $B, f^{\prime} \cap$ obviously reduces filtration. Thus if both $\alpha$ and $\beta$ are $1-1$ or onto, then $F$ is $1-1$ or onto respectively. In particular if both $\alpha$ and $\beta$ are isomorphisms so is $F$. The original requirements for $F$ to be a morphism in $\mathscr{B} \mathscr{C} \mathscr{A}$ provide the commutativity of the following two diagrams, which makes it clear that $F^{-1}$ is also a morphism in $\mathscr{B} \mathscr{A} \mathscr{A}:$

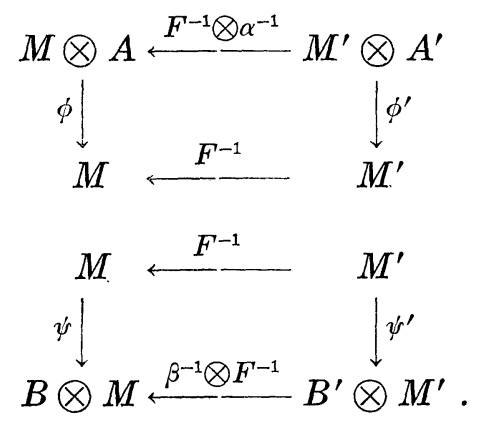

Using Proposition 2 for $F^{-1}$, there exists a degree 0 linear map $g: B^{\prime} \rightarrow A$ such that $F^{-1}=g \cap$. Composition gives us the following equalities:

$$
1=F^{-1} F=g \cap f \cap .
$$

Similarly $1=F F^{-1}=f \cap g \cap$. If $\alpha=1, \beta=1$ the above reduces to:

$$
(g \cup f) \cap=1 \text { or } g \cup f=\mathscr{E} .
$$

And $g \cup f=f \cup g$ is now obvious. Summarizing the above we get:

Proposition 3. Let $(F, \beta, \alpha): M \rightarrow M^{\prime}$ be morphism in $\mathscr{B} \mathscr{L} \mathscr{\mathscr { A }}$. If $\alpha, \beta$ are isomorphisms then $F^{-1}$ exists and is a morphism in $\mathscr{B} \mathscr{C} \mathscr{A}$. If $\alpha=1, \beta=1, F=f \cap$ and $F^{-1}=g \cap$ then $g \cup f=\mathscr{O}$ and $f \cup g=g \cup f$. 
It is now natural to introduce the following notation:

DEFINITION 2. (i) Let T.T. denote the category whose objects are of the form $B \otimes A$ and whose morphism are $F=(\beta, \alpha, f): B \otimes A \rightarrow$ $B^{\prime} \otimes A^{\prime}$ such that $F=f \cap$ where $A, \alpha \in \mathscr{A}, B, \beta \in \mathscr{B}$ and $f: B \rightarrow A^{\prime}$ is a degree 0 linear map. (ii) Let D.T.T. denote the above category such that objects possess a differential $D=D_{0}+x \cap$ where $D_{0}$ is the usual differential induced from the differential of $A$ and $B$ and $x: B \rightarrow A$ is a degree -1 linear map. We already denoted this object by $B \otimes_{x} A$. The morphisms in the category are those of T.T. with the extra requirement that they commute with the differentials.

REMARK 3. The requirement for $D=D_{0}+x \cap$ to be a differential is equivalent to

$$
d x+x d+x \cup x=0 .
$$

If $(\beta, \alpha, f): B \bigotimes_{x} A \rightarrow B^{\prime} \bigotimes_{x^{\prime}} A^{\prime}$ is a map in D.T.T. then $f$ has to satisfy the equation:

$$
d f-f d=f \cup \alpha x-x^{\prime} \beta \cup f,
$$

We have established in this section:

THEOREM 1. The category of bundles $\mathscr{B} \mathscr{A} . \mathscr{A}$ is the skeleton of the category of twisted tensor products D.T.T.

3. A Lie algebra for a bundle. By Remark 2 it is not hard to be convinced that all maps of degree $k$, which respect the structure of the objects in $\mathscr{B} \mathscr{K} \cdot \mathscr{A}$, i.e., make diagrams (2) and (3) commutative, are precisely the maps of the form $\tau \cap$, where $\tau$ is a linear map of degree $k$. We exploit these functions in our construction of a differential algebra from which we obtain the associated desired Lie algebra.

Let $B \in \mathscr{B}, A \in \mathscr{\mathscr { L }}$,

$$
\operatorname{Hom}^{k}(B, A)=\{\text { all linear maps of degree } k\},
$$

and

$$
L(B, A)^{k}=\left\{\tau \cap \mid \tau \in \operatorname{Hom}^{-k}(B, A)\right\} \quad k \in Z .
$$

We define multiplication in $L$ as composition of maps, which provides us with an associative algebra structure with unit, because composition turns out to be the cup product of the defining linear 
maps:

$$
\tau_{2} \cap \tau_{1} \cap=\left(\tau_{2} \cup \tau_{1}\right) \cap
$$

The tensor product differential $D_{0}$ of $B \otimes A$ induces a natural differential on $L$, and makes it into a differential graded algebra, where:

$$
\delta(\tau \cap)=\left[D_{0}, \tau \cap\right]=\left(d \tau-(-1)^{k} \tau d\right) \cap|\tau|=k .
$$

It is straight forward to check that $\delta^{2}=0$ and that it is a derivation.

We concentrate on the differential Lie algebra associated with the above algebra having in mind applications of algebraic geometry techniques. The multiplication expressed explicitly is:

$$
\left[\tau \cap, \tau^{\prime} \cap\right]=\left(\tau \cup \tau^{\prime}-(-1)^{k l} \tau^{\prime} \cup \tau\right) \cap,
$$

where $|\tau|=k\left|\tau^{\prime}\right|=l$. Because of the naturality of the definition we have:

THEOREM 2. There is a functor from $\mathscr{B} \times \mathscr{A}$ to the category of differential graded Lie algebras, contravariant in the first variable and covariant in the second, which assigns to the object $(B, A)$ the differential Lie algebra $L(B, A)$. If $\beta: B^{\prime} \rightarrow B \in \mathscr{B}, \alpha: A \rightarrow$ $A^{\prime} \in \mathscr{A}$, then we get a differential Lie algebra homomorphism $L(B, A) \rightarrow L\left(B^{\prime}, A^{\prime}\right)$ defined by $\tau \cap \rightarrow \alpha \tau \beta \cap$.

4. Classification of bundles and twisted tensor products.

Definition 3. (i) Two $(B, A)$ bundles $M$ and $M^{\prime}$ are equivalent if there is a $(B, A)$ boundle isomorphism $F: M \rightarrow M^{\prime}$ in $\mathscr{B} \mathscr{R} \cdot \mathscr{A}$.

(ii) Two twisted tensor products $B \bigotimes_{x} A$ and $B \bigotimes_{x^{\prime}} A$ are equivalent if there is a map $1+f \cap: B \otimes_{x} A \rightarrow B \boldsymbol{\bigotimes}_{x^{\prime}} A \in$ D.T.T.

Using the information given by Theorem 1 we get that the equivalence classes of $(B, A)$ bundles and equivalence classes of twisted tensor products of the form $B \otimes_{x} A$ with $B$ and $A$ fixed, are isomorphic. Thus we will be concerned with twisted tensor products. We show next that maps of the form $F=1+\cap f$ which are morphisms in T.T. act on the elements of D.T.T. under consideration, and form the desired equivalence classes.

Let $F=1+f \cap, F^{-1}=G=1+g \cap$ and $D=D_{0}+x \cap$ a differential. We prove that $G D F$ is also a differential of $B \otimes A$, and turns $B \otimes A$ into an element of D.T.T.. We also find the explicit expression of $G D F$.

Consider the diagram: 


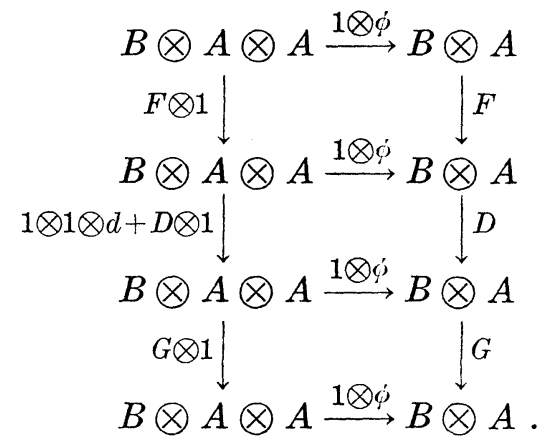

Each of the small rectangles commutes, which leads to the commutativity of the outer rectangle. This and an analogous argument provides us with the following commutative diagrams:

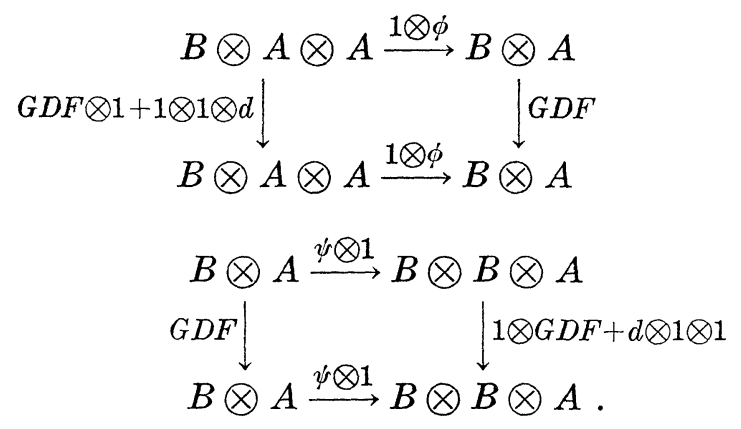

Thus $(B \otimes A, G D F)$ is an element of $\mathscr{B} \mathscr{C} \mathscr{C}$. Applying Proposition 1 we get that $G D F=D_{0}+x^{\prime} \cap$. We compute $x^{\prime}$ in terms of $x, f$ and $g$ :

$$
\begin{aligned}
G D F= & (1+g \cap)\left(D_{0}+x \cap\right)(1+f \cap) \\
= & D_{0}+(x+x \cup f+g \cup x+g \cup x \cup f+d f+g d+g d \cup f \\
& +g \cup d f) \cap .
\end{aligned}
$$

Note that this equivalence relation is actually not made explicit, since $g$ is not explicit. We overcome this in the next section via the "log" function. We have established:

THEOREM 3. The collection of isomorphisms $F=(1+\cap f)$ : $B \otimes A \rightarrow B \otimes A \in \mathrm{T} . \mathrm{T}$. acts on the collection of objects $(B \otimes A$. $\left.D=D_{0}+x \cap\right) \in$ D.T.T. via conjugation of the differential, i.e., $(B \otimes A, D) \rightarrow\left(B \otimes A, F^{-1} D F\right)$. The orbits of this action form the equivalence classes of $(B, A)$ twisted tensor products.

REMARK 4. Note that if $D$ in the above discussion is replaced by $\tau \cap$ with $\tau \in \operatorname{Hom}^{k}(B, A)$ then from similar considerations and 
Remark 2 above we get that $G(\tau \cap) F$ is also of the form $\tau^{\prime} \cap, \tau^{\prime} \in$ $\operatorname{Hom}^{k}(B, A)$.

5. The classifying moduli space of twisted tensor products. At this point the connection between the Lie algebra $L(B, A)$, defined in $\S 3$, and the classification of $(B, A)$ bundles or $(B, A)$ twisted tensor products does not seem remote any more. Next we clarify this relation.

As already pointed out in $\S 2, D_{0}+x \cap$ is a differential in D.T.T. only if $x$ is integrable, or more explicitly:

Definition 4. The variety of elements of $L(B, A)^{1}$ which correspond to differentials of elements in D.T.T. will be denoted by:

$$
I(B, A)=\left\{x \cap \in L(B, A)^{1} \mid\left[D_{0}, x \cap\right]+\frac{1}{2}[x \cap, x \cap]=0\right\} .
$$

Any $f \cap \in L(B, A)$ applied to an element $b \otimes a \in B \otimes A$ reduces the $B$-degree filtration of the element obtained, and makes the action of $L(B, A)^{0}$ on $L(B, A)$ complete. This justifies the following definitions on elements of $L(B, A)^{0}$ and morphisms $B \otimes A \rightarrow B \otimes A \epsilon$ T.T. as the summations turn out to be finite when applied on elements of $B \otimes A$. We should also point out that at this stage we have to restrict ourselves to a characteristic 0 field, since next we are going to employ fractions.

Definition 5. (i) Let $f \cap \in L(B, A)^{0}$. Then

$$
\begin{aligned}
\exp (f \cap)= & \sum_{n=0}^{\infty} \frac{(f \cap)^{n}}{h !}=1+f \cap+\frac{1}{2 !}(f \cup f) \cap+\cdots \\
& +\frac{1}{n !}(f \cup \cdots \cup f) \cap+\cdots
\end{aligned}
$$

(ii) Let $F=1+f \cap: B \otimes A \rightarrow B \otimes A \in \mathrm{T} . \mathrm{T} . . \quad$ Then

$$
\begin{gathered}
\log (1+f \cap)=\sum_{n=1}^{\infty} \frac{(f \cap)^{n}}{n}=f \cap-\frac{1}{2}(f \cup f) \cap+\cdots \\
\pm \frac{1}{n}(f \cup \cdots \cup f) \cap \pm \cdots
\end{gathered}
$$

REMARK 5. We make the following observations about the functions just defined:

(i) $\exp (f \cap)$ is an isomorphism: $B \otimes A \rightarrow B \otimes A \in \mathrm{T}$.T.

(ii) $\log (1+f \cap) \in L^{0}(B, A)$

(iii) $\exp (\log (1+f \cap))=1+f \cap$ 
(iv) $\log (\exp (f \cap))=f \cap$

(v) $\exp ((-f) \cap)=\exp (-f \cap)=\exp (f \cap)^{-1}$.

Let $f \cap \in L(B, A)^{0}$. Then direct computation yields the following:

$$
\begin{aligned}
\exp [ & f \cap, \quad]\left(D_{0}+x \cap\right)=\sum_{n=0}^{\infty} \frac{[f \cap,]^{n}\left(D_{0}+x \cap\right)}{n !} \\
& =\left(\sum_{n=0}^{\infty} \frac{(f \cap)^{n}}{n !}\right)\left(D_{0}+x \cap\right)\left(\sum_{n=0}^{\infty} \frac{(-f \cap)^{n}}{n !}\right) \\
& =\exp (f \cap)\left(D_{0}+x \cap\right) \exp (-f \cap) .
\end{aligned}
$$

Note that $\exp (f \cap)=F: B \otimes A \rightarrow B \otimes A$ is an isomorphism in T.T.. Using Theorem 3 and Remark 4 we obtain an operation of $L(B, A)^{0}$ on $L(B, A)$ via exp. It is also straight forward to check that $L(B, A)^{0}$ operates on $I(B, A)$. Since exp is an isomorphism from the set $L(B, A)^{0}$ to the set of all morphisms: $B \otimes A \rightarrow B \otimes A \in \mathrm{T}$.T., we have proved:

THEOREM 4. The set of equivalence classes of $(B, A)$ bundles, or equivalence classes of $(B, A)$ twisted tensor products is isomorphic to the moduli space $E(B, A)=I(B, A) / \exp L(B, A)^{0}$, where the action is via conjugation.

6. Computation of $E(B, A)$. At this stage the computation of the equivalence classes reduced to calculating a moduli space associated to a Lie algebra. Thus we can apply algebraic geometry deformation theory to the later. For the convenience of the reader we simplify the notation and state some of the relevant theorems [6].

We denote by $L$ the Lie algebra $L(B, A)$, by $I$ the integrable elements $I(B, A)$, and by $E=E(L)=I / \exp L^{0}$ the moduli space.

THEOREM A. If $f: K \rightarrow L$ is a map of differential graded Lie algebras which are decreasingly filtered, complete and bounded from above in each degree such that $f$ induces

$$
\begin{aligned}
& \text { a monomorphism } H^{2}(K) \longrightarrow H^{2}(L) \\
& \text { an isomorphism } H^{1}(K) \longrightarrow H^{1}(L)
\end{aligned}
$$

and

$$
\text { an epimorphism } H^{\circ}(K) \longrightarrow H^{0}(L),
$$

then $f$ induces an isomorphism from $E(K)$ to $E(L)$. Under these hypotheses we say that $K$ and $L$ are homotopy equivalent in degree 1.

THEOREM B. If $\operatorname{dim} H^{1}(L)$ is finite, then $E$ has a representation $E=W / F$ where: 
(i) $W$ is a formal variety defined by $n$ formal power series equations $f_{j}\left(x_{1}, \cdots, x_{m}\right)=0$ where $m=\operatorname{dim} H^{1}(L)$ and $n=\operatorname{dim} H^{2}(L)$.

(ii) $F \rightarrow W \times W$ is an equivalence relation in which the orbit of the origin in $W$ is trivial.

(iii) Any versal $I$ is equivariantly isomorphic to $W \times Z$, where $Z$ is some affine space with trivial equivalence relation.

(iv) If $L \cong H(L)$ in degree 1 , then $W$ is the pure quadratic variety $\left\{l \in H^{1}(L) \mid[l, l]=0\right\}$ and $E=W / \exp H^{0}(L)$.

The above citation leads us to compute $H^{*}(L(B, A))$. Define the following function:

$$
S: L(B, A) \longrightarrow \operatorname{Hom}^{-*}(B, \bar{A})
$$

by $S(f \cap)=f$ for any $f \cap \in L(B, A)^{k}$. If we examine the associative algebras of $L(B, A)$ and of $\mathrm{Hom}^{-*}(B, \bar{A})$, where the laters algebra structure is the cup product, we realize that $S$ is an algebra homomorphism. With the usual differential given to $\operatorname{Hom}^{*}(B, \bar{A})$, i.e.,

$$
D(f)=d f-(-1)^{k} f d \quad|f|=k,
$$

$S$ is also differential. Thus $S$ can be considered as a differential Lie algebra isomorphism. We have:

$$
\begin{aligned}
H^{i}(L(B, A)) & =H\left(\operatorname{Hom}^{-*}(B, \bar{A})\right)=H_{-i}(\operatorname{Hom}(B, \bar{A})) \\
& =\operatorname{Hom}_{-i}(H(B), \bar{H}(A))=\left(H^{*}(B) \otimes \bar{H}_{*}(A)\right)_{-i}
\end{aligned}
$$

where $\bar{H}_{*}(A)$, the augmentation ideal is of finite type.

7. Twisted cartesian and tensor products. In this section we attempt to relate twisted cartesian products to twisted tensor products. Whatever we achieve here, will be used in the next section to connect the classification of twisted cartesian products or actually principal fibrations to the classification of twisted tensor products.

To make this paper self contained, we recall briefly some concepts from [3]. We use the same notation for both simplicial sets and maps and their normalized chain complex and chain map respectively.

The simplicial set $B \times{ }_{x} A$ is called a twisted cartesian product where the base $B$ is a simplicial set, $A$ is a simplicial group and $x: B \rightarrow A$ is a degree -1 twisting function. A map $F=(\beta, \alpha, f)$ : $B \times{ }_{x} A \rightarrow B^{\prime} \times{ }_{x^{\prime}} A^{\prime}$ defined by $F(b, a)=(\beta(b), f(b) \alpha(a))$, where $\alpha$ : $A \rightarrow A^{\prime}$ is a simplicial homomorphism, $\beta: B \rightarrow B^{\prime}$ is a simplicial map and $f: B \rightarrow A^{\prime}$ is a function which keeps $F$ simplicial. If $f$ is the constant function to the unit we denote it by $e$ and say that $F$ is untwisted. We denote the resulting category by T.C.. Let $\nabla: B \otimes$ 
$A \rightarrow B \times A, f: B \times A \rightarrow B \otimes A, \dot{\phi}: B \times A \rightarrow B \times A$ be the Eilenberg Zilber theorem maps which satisfy: $f F=1, \phi V=0, f \phi=0$, $\phi \dot{\phi}=0$ and $\nabla f^{\prime}=1+d \dot{\phi}+\dot{\phi} d$. Gugenheim defined a functor $\Gamma^{\prime}:$ T.C. $\rightarrow$ D.T.T. by perturbing the E.Z. maps, where T.C'. is T.C. with the twisted morphisms deleted. For each twisting function $x$ he defined a twisting cochain which we also denote by $x$. The functor $\Gamma^{\prime}$ is thus defined by $\Gamma^{\prime}\left(B \times{ }_{x} A\right)=B \otimes{ }_{x} A, \Gamma^{\prime}(\beta, \alpha, e)=\beta \otimes \alpha$. The twisted E.Z. maps satisfy the original equations and carry the same notation, i.e., $\nabla: B \otimes{ }_{x} A \rightarrow B \times{ }_{x} A$.

We extend $\Gamma^{\prime}$ to $I^{\prime \prime}$ : T.C. $\rightarrow$ D.T.T. by keeping $\Gamma^{\prime \prime}\left(B \times{ }_{x} A\right)=$ $\Gamma^{\prime \prime}\left(B \times{ }_{x} A\right)$ and setting $\Gamma^{\prime \prime}(F)=f^{\prime} F F: B \otimes{ }_{x} A \rightarrow B^{\prime} \otimes{ }_{x^{\prime}} A^{\prime}$.

To justify our construction we have to show:

(i) $\Gamma^{\prime \prime}$ is an extension of $\Gamma^{\prime}$.

(ii) $\Gamma^{\prime \prime}(F)$ is a morphism in D.T.T.

(iii) $\Gamma^{\prime \prime}$ is a functor.

(i) is easy to show, (ii) is lengthy and (iii) we are not able to show. However we take care of this requirement by replacing $\Gamma^{\prime \prime}$ by $T$.

(i) We have to show that $I^{\prime \prime}(\beta, \alpha, e)=\beta \otimes \alpha$. In the construction of the twisted $\Gamma$ it was kept natural for untwisted maps, i.e. $(\beta>\alpha) \Gamma=\Gamma^{\prime}(\alpha \otimes \beta)$. Thus $\Gamma^{\prime \prime}(\beta, \alpha, e)=f^{\prime}(\beta \times \alpha) \Gamma=f^{\prime} \Gamma^{\prime}(\beta \otimes \alpha)=$ $\beta \otimes \alpha$.

(ii) Let $F: B \times{ }_{x} A \rightarrow B^{\prime} \times{ }_{x} \cdot A^{\prime}$. Consider the following diagram:

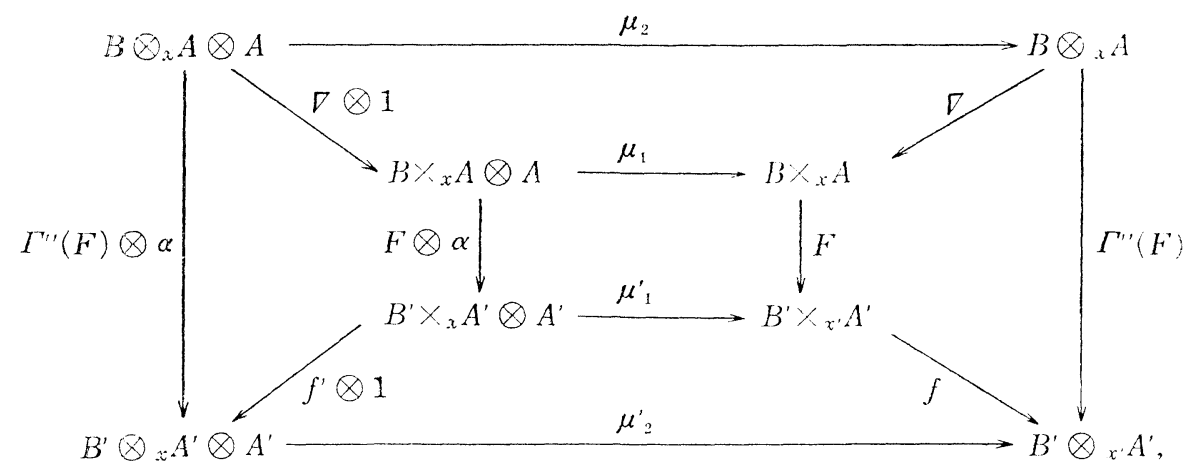

where $\mu_{2}$ is the extended module structure map and $\mu_{1}$ is the composition of the appropriate untwisted map $V$ and the group multiplication of $A$ :

$$
B \times{ }_{x} A \otimes A \longrightarrow B \times{ }_{x} A \times A \longrightarrow B \times{ }_{x} A .
$$

The commutativity of the upper and lower trapezoides is proved in [3|. The two remaining trapezoides commute by the definition of 
$\Gamma^{\prime \prime}$. There is left to prove the commutativity of the inner rectangle.

Consider the following diagram:

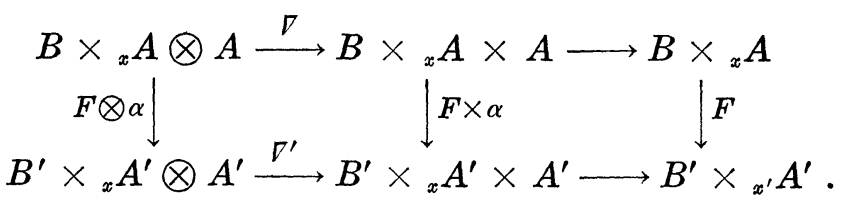

The right hand side rectangle commutes trivially. The left hand side commutes because of the naturality of $\nabla$ in the untwisted case. Some diagram chaining gives us the commutativity of the outer rectangle in (11).

Next consider the diagram:

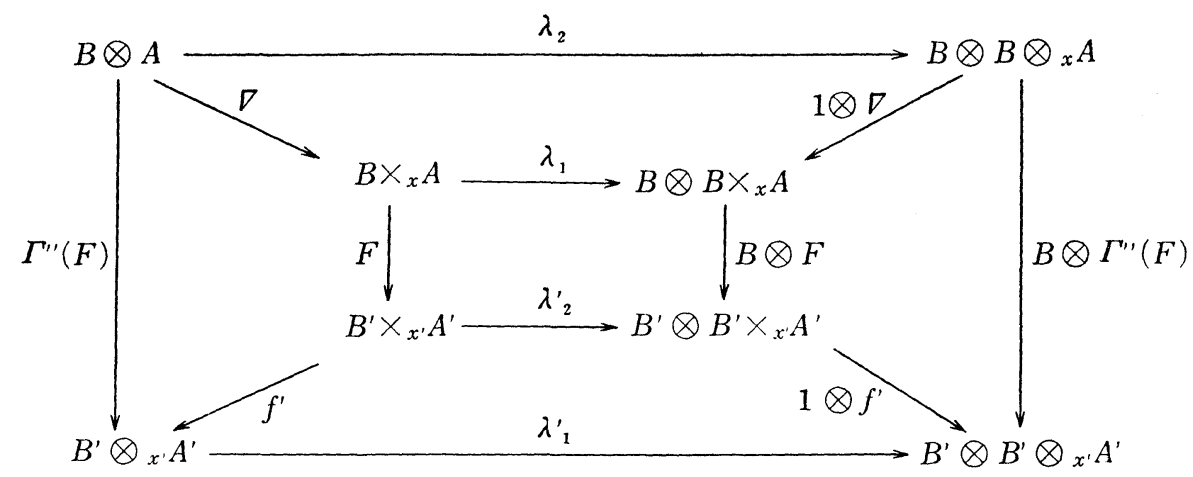

where $\lambda_{2}$ is the extended comodule structure map, and $\lambda_{1}$ is induced by the diagonal map of $B$ and the appropriate untwisted E.Z. map $f$. The commutativity of the exterior rectangle is obtained similarly to the previous case. We conclude that $\Gamma^{\prime \prime}(F)$ is a morphism in $\mathscr{B}$ Lll. $\mathscr{A}$, i.e., can be represented by a morphism in D.T.T.

(iii) In checking if $\Gamma^{\prime \prime}$ is a functor we find that $\Gamma^{\prime \prime}$ behaves well only when one of the morphisms composed is untwisted:

$$
\begin{gathered}
\Gamma^{\prime \prime}((\beta, \alpha, e) F)=f^{\prime \prime}(\beta \times \alpha) F \nabla=(\beta \otimes \alpha) f^{\prime} F \nabla=\Gamma^{\prime \prime}(\beta, \alpha, e) \Gamma^{\prime \prime}(F), \\
\Gamma^{\prime \prime}(F(\beta, \alpha, e))=f^{\prime \prime} F(\beta \times \alpha) \nabla=f^{\prime \prime} F \nabla^{\prime}(\beta \otimes \alpha)=\Gamma^{\prime \prime}(F) \Gamma^{\prime \prime}(\beta, \alpha, e) .
\end{gathered}
$$

However if $F$ and $F^{\prime}$ are twisted we get:

$$
\begin{aligned}
& \Gamma^{\prime \prime}\left(F^{\prime} F\right)=f^{\prime \prime} F^{\prime} F \nabla \\
& \Gamma^{\prime \prime}\left(F^{\prime}\right) \Gamma^{\prime \prime}(F)=f^{\prime \prime} F^{\prime} \nabla^{\prime} f^{\prime} F V
\end{aligned}
$$

which do not have to coincide. We make some identifications of morphisms in D.T.T. Let $G, G^{\prime}, G_{i}, G_{i}^{\prime}$ be morphisms in D.T.T. and $F_{i}$ morphisms in T.C.. Define on elementary equivalence by: 


$$
G_{2} f F_{2} \nabla f F_{1} \nabla G_{1} \sim G_{2} f F_{2} F_{1} \nabla G_{2} .
$$

An equivalence $G \cong G^{\prime}$ takes place if there exists a sequence of elementary equivalences:

$$
G=G_{1}^{\prime} \sim G_{2}^{\prime} \sim \cdots \sim G_{n}^{\prime}=G^{\prime} .
$$

This relation defines a quotient category of D.T.T., which we denote by ho.D.T.T.. We proved:

THEOREM 5. There exists a functor $\Gamma^{\prime \prime}:$ T.C. $\rightarrow$ ho.D.T.T. defined as follows:

$$
\begin{aligned}
& \Gamma\left(B \times{ }_{x} A\right)=B \otimes{ }_{x} A, \\
& \Gamma(F)=[f F \nabla],
\end{aligned}
$$

where [ ] denotes the equivalence class in ho.D.T.T. of a morphism of D.T.T..

8. On the classification of fibrations. It is well known that Serre fibrations can be represented by Kan fibrations and they in turn to twisted cartesian products [1,5]. Gugenheim [3] following Brown [2] associated to the later a twisted tensor product going first through bundles. There is no loss of generality by treating only principal objects, as passing to the none principal case involves just tensoring with the fiber over the group.

We try to use the classification of twisted tensor products for the classification of principal fibrations via the functor $\Gamma$, introduced in the previous section. Observe that the category of principal fibrations is the skeleton of the category T.C.. Thus the equivalence classes of $(B, A)$ principal fibrations are isomorphic to the equivalence classes of $(B, A)$ twisted cartesian products. Thus the equivalence classes of $(B, A)$ principal fibrations is approximated by the equivalence classes of the $(B, A)$ bundles in ho.T.T.. This relationship becomes precise in the following situation:

Consider the $\left(S^{4}, S^{3} \times S^{3}\right)$ principal fibrations, over the rationals $Q$. Using the computations of $\S 6$ we get:

$$
\begin{gathered}
H^{0}(L)=H^{2}(L)=0 H^{1}(L)=Q \oplus Q, \\
\Longrightarrow E\left(S^{4}, S^{3} \times S^{3}\right)=Q \oplus Q .
\end{gathered}
$$

This is compatible with the fact:

$$
\pi_{4}\left(B\left(S^{3} \times S^{3}\right)\right)=\pi_{4}\left(B S^{3} \times B S^{3}\right)=Z \oplus Z .
$$




\section{REFERENCES}

1. M. G. Barratt, V. K. A. M. Gugenheim and J. C. Moore, On semi-simplicial fibre bundles, Amer. J. Math., 81 (1959), 639-657.

2. E. H. Brown, Jr., Twisted tensor products I, Ann. of Math., (2), 69 (1959), 223-246.

3. V. K. A. M. Gugenheim, On the chain-complex of a fibration, Illinois J. Math., 16 (3) (1972), 398-414.

4. D. Husemoller, J. C. Moore and J. Stasheff, Differential homological algebra and homogeneous spaces, J. of Pure and Applied Algebra, 5 (2) (1974), 113-185.

5. J. P. May, Simplicial objects in algebraic topology, Van Nostrand Mathematical studies, no' 11.

6. M. Schlessinger and J. Stasheff, Deformation theory and rational homotopy type, (preprint).

Received December 19, 1980 and in revised form April 16, 1981.

UNiversity of NoRth Carolina

Chapel HILl, NC 27514 


\section{PACIFIC JOURNAL OF MATHEMATICS}

\section{EDITORS}

DONALD BABBITT (Managing Editor)

University of California

Los Angeles, CA 90024

Hugo RossI

University of Utah

Salt Lake City, UT 84112

C. C. Moore and Arthur Agus

University of California

Berkeley, CA 94720
J. DugundJI

Department of Mathematics

University of Southern California

Los Angeles, CA 90007

R. FinN and J. MiLgRAM

Stanford University

Stanford, CA 94305

\section{ASSOCIATE EDITORS}
R. ARENS
E. F. BECKENBACH
B. H. NeumanN
F. WOLF
K. YoSHIDA

\section{SUPPORTING INSTITUTIONS}

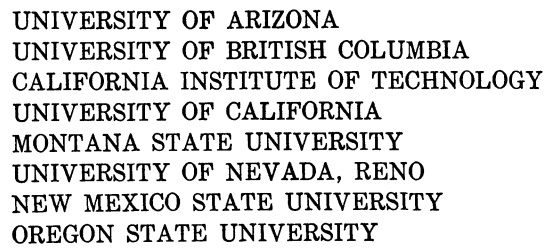

UNIVERSITY OF ARIZONA

UNIVERSITY OF BRITISH COLUMBIA CALIFORNIA INSTITUTE OF TECHNOLOGY UNIVERSITY OF CALIFORNIA MONTANA STATE UNIVERSITY

UNIVERSITY OF NEVADA, RENO NEW MEXICO STATE UNIVERSITY OREGON STATE UNIVERSITY

\author{
UNIVERSITY OF OREGON \\ UNIVERSITY OF SOUTHERN CALIFORNIA \\ STANFORD UNIVERSITY \\ UNIVERSITY OF AAWAII \\ UNIVERSITY OF TOKYO \\ UNIVERSITY OF UTAH \\ WASHINGTON STATE UNIVERSITY \\ UNIVERSITY OF WASHINGTON
}

The Supporting Institutions listed above contribute to the cost of publication of this Journal, but they are not owners or publishers and have no responsibility for its content or policies,

Mathematical parers intended for publication in the Pacific Journal of Mathematics should be in typed form or offset-reproduced, (not dittoed), double spaced with large margins. Please do not use built up fractions in the text of the manuscript. However, you may use them in the displayed equations. Underline Greek letters in red, German in green, and script in blue. The first paragraph or two must be capable of being used separately as a synopsis of the entire paper. Please propose a heading for the odd unmbered pages of less than 35 characters. Manuscripts, in triplicate, may be sent to any one of the editors. Please classify according to the scheme of Math. Reviews, Index to Vol. 39. Supply name and address of author to whom proofs should be sent. All other communications should be addressed to the managing editor, or Elaine Barth, University of California, Los Angeles, California, 90024 .

50 reprints to each author are provided free for each article, only if page charges have been substantially paid. Additional copies may be obtained at cost in multiples of 50 .

The Pacific Journal of Mathematics is issued monthly as of January 1966, Regular subscription rate: $\$ 114.00$ a year (6 Vol., 12 issues). Special rate: $\$ 57.00$ a year to individual members of supporting institution.

Subscriptions, orders for numbers issued in the last three calendar years, and changes of address shoud be sent to Pacific Journal of Mathematics, P.O. Box 969, Carmel Valley, CA 93924, U.S.A. Old back numbers obtainable from Kraus Periodicals Co., Route 100, Millwood, NY 10546.

\section{PUBLISHED BY PACIFIC JOURNAL OF MATHEMATICS, A NON-PROFIT CORPORATION}

Printed at Kokusai Bunken Insatsusha (International Academic Printing Co., Ltd.). 8-8, 3-chome, Takadanobaba, Shinjuku-ku, Tokyo 160, Japan.

Copyright (C) 1982 by Pacific Journal of Mathematics Manufactured and first issued in Japan 


\section{Pacific Journal of Mathematics}

Vol. 100, No. $2 \quad$ October, 1982

Kenneth F. Andersen, On the transformation of Fourier coefficients of

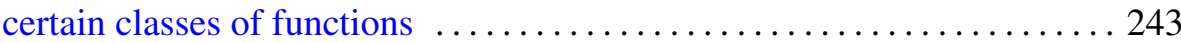

Steven Albert Bleiler, Realizing concordant polynomials with prime knots

Reinhard Bürger, Functions of translation type and solid Banach spaces of functions

Ulrich Daepp, The saturation of $k$-analytic rings and topological equivalence of associated analytic set germs .................. 271

Persi W. Diaconis and David Amiel Freedman, On the maximum difference between the empirical and expected histograms for sums . . . 287

David Amiel Freedman, On the maximum of scaled multinomial

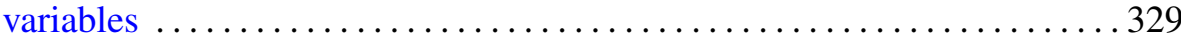

Persi W. Diaconis and David Amiel Freedman, On the difference between the empirical histogram and the normal curve, for sums. II ......... 359

Persi W. Diaconis and David Amiel Freedman, On the mode of an

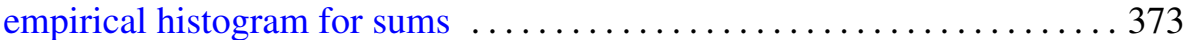

Jutta Hausen, Supplemented modules over Dedekind domains 387

Elyahu Katz, A moduli representation for the classification of twisted tensor

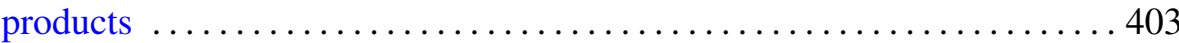

H. C. Madhekar and N. K. Thakare, Biorthogonal polynomials suggested by the Jacobi polynomials

Ted R. Pettis, Collections of covers of metric spaces 425

Ryōtarō Satō, Maximal functions for a semiflow in an infinite measure space

Michael Jay Stob, Invariance of properties under automorphisms of the lattice of recursively enumerable sets 